\title{
ESTUDIO EXPLORATORIO DE LAS INTERACCIONES MENTALES DE LOS ESTUDIANTES DE SEXTO GRADO SOBRE EL ENTENDIMIENTO CONCEPTUAL DE MULTIPLICACIÓN ${ }^{1}$
}

\section{EXPLORATORY ANALYSIS OF MENTAL INTERACTIONS IN STUDENTS OF SIXTH DEGREE ON THEIR CONCEPT UNDERSTANDING OF MULTIPLICATION}

\author{
Roberto Figueroa Molina² \\ roberfigue@hotmail.com \\ Carlos Utria Echeverría ${ }^{2}$ \\ Rafael Colpas Castillo2 \\ Antonio Araújo Pereira ${ }^{2}$
}

\begin{abstract}
This work constitutes a descriptive case análisis intended to explore how students of mathematical sixth grade build and internalize the concept of multiplication from their beginanig in the formal education. It olso reviews the process they apply to elaborate their real multiplication concept.
\end{abstract}

Key Words: mental interactions, exploratory study, multiplication, interview, conceptualization.

\section{RESUMEN}

La presente investigación constituye un estudio de caso de tipo descriptivo. Su propósito consistió en explorar cómo los estudiantes de sexto (6to) grado, construyen o interiorizan el concepto de multiplicación desde los inicios de su educación formal, junto con los procesos para elaborar la concepción sobre el concepto de multiplicar que actualmente poseen.

Palabras Clave: interacciones mentales, estudio exploratorio, multiplicación, entrevista reconciliadora, conceptualización.

\footnotetext{
${ }_{1}^{1}$ Parte de este trabajo fue presentado en el Seminario sobre Investigación Descriptiva. Universidad de Puerto Rico. 1997.

2 Profesores de la Universidad del Atlántico. Miembros del Grupo "GECIT" reconocido por Colciencias.
} 


\section{Presentación}

Documentos recientes relacionados con la reforma educativa en matemáticas (Concilio Nacional de Maestros de Matemáticas, NCTM, 1989; Concilio Nacional de Investigaciones, 1989), han hecho un llamado para minimizar el énfasis en los cómputos y hechos y brindar un mayor énfasis al entendimiento de conceptos (comprensión), solución de problemas, aplicaciones y comunicación de las ideas matemáticas, preconceptos, interacciones mentales y el desarrollo en los aprendices de un entendimiento matemático comprensivo y con la suficiente flexibilidad para aplicar estos conceptos en una amplia gama de problemas del mundo real (Simon and Blume, 1994).

Como parte de esta propuesta, es necesario reconocer y analizar el concepto de multiplicación como un aspecto de razonamiento cuantitativo que Thompson (1989) define como un problema de pensamiento sobre una situación de razonamiento multiplicativo (multiplicación). ¿Cómo el individuo adquiere este concepto?, ¿cómo lo internaliza, desarrolla, metacogniza y lo aplica?

Cuando los estudiantes son expuestos a experiencias que convergen con sus valores y conocimientos con los cuales pueden hacer uso de los niveles de representación que poseen sobre la multiplicación (Barody 1987; Siffe 1989 y Kouba 1989), estas concepciones pueden alterar el proceso formal del aprendizaje de la multiplicación. Esto se debe a que pueden aplicar ciertos conocimientos sobre la multiplicación, y así lo hacen, a través de los niveles de representación de la multiplicación antes de conocer el concepto o los símbolos formales de la multiplicación (Kouba and Franklin, 1995).

Por lo tanto, este aspecto del razonamiento matemático puede solaparse con otros estudios del campo científico que abordan las ideas previas, preconcepciones y errores conceptuales. El problema ha sido estudiado en otros aspectos matemáticos como el de fracción (Melun, 1994), multiplicación y división (Kouba and Franklin 1995; Clark, 1993; Clark, 1996).

La multiplicación es un concepto que se desarrolla lentamente a través de la vida escolar del estudiante. Requiere de bases conceptuales y operativas de la suma, que a su vez se cimenta sobre los conceptos de conjunto, relaciones y funciones. Esto sugiere que, con el tiempo, el estudiante pasa por los procesos de asimilación y acomodación del concepto en sus esquemas mentales, pero intercalando los escenarios en los cuales se da la experiencia. En la medida que el estudiante pasa de un grados escolares a otro, se incrementan sus conocimientos y la concepción sobre la multiplicación. Ello implica que un estudiante de primer grado conoce menos del concepto que uno de quinto, y así sucesivamente hasta el grado $12 \mathrm{mo}$.

A través de este estudio, se pretende describir cómo el estudiante de sexto (6to) grado ha construido o internalizado el concepto de multiplicación, desde su inicio en los estudios formales y el proceso que ha seguido para elaborar la percepción sobre el concepto de multiplicar que hoy posee.

Para recopilar la información sobre este aspecto, se utilizó como instrumento la entrevista reconciliado- ra (Figueroa, 1995), la cual permite globalizar todas las respuestas posibles, a través de pre- 
guntas que exploran el desarrollo continuo y gradual del concepto de multiplicación de los estudiantes de sexto grado.

\section{Planteamiento del problema}

Muchos de los aspectos o características que se han estudiado con relación a las concepciones que tenemos los humanos de ciertos fenómenos u objetos y sus interacciones cognoscitivas, aún después de haber recibido instrucción prosiguen vigentes, aunque el esfuerzo por modificarlas continúa. La exploración sobre la percepción que poseen los estudiantes, particularmente los de sexto grado, sobre el concepto de multiplicación está permeada por una serie de factores específicos tales como:

- La multiplicación generalmente se le presenta a los estudiantes en el segundo grado y proyectada simplemente como una forma rápida para hacer suma repetitivas (Fennell, Reys and Webb, 1991).

- Los niños suman en lugar de multiplicar (Hart 1981; Kamii and Livingston, 1994).

- Los niños con algún tipo de dificultad en los cómputos, a menudo presentan problemas con el significado de multiplicación (Lindguist 1989; Piaget 1983/ 1987).

- Cuando los niños no conocen un "producto", no pueden computarlo a partir de lo que conocen. Esta dificultad contrasta con la facilidad de los niños en calcular la suma desconocida. Ejemplo: cuando un niño conoce que $6 \times 6=36$, esto no ayuda a muchos niños a multiplicar 7 X6 (Kamii and Livingston, 1994).
- Estrategias metodológicas de la enseñaza centrada en la repetición, memorización y evaluación acumulativas. Autores de textos de matemáticas ignoran las deficiencias conceptuales de sus contenidos, lo cual disminuye la posibilidad de aplicar procesos de enseñanza y aprendizaje significativos (Figueroa, 1996).

- Enseñanza focalizada en la solución de ejercicios mediante algoritmos, convirtiendo los estudiantes en simples mecánicos, restándole importancia a los procesos descriptivos y explicativos que facilitan en el alumno una mejor interpretación del concepto de multiplicación (Figueroa, 1996).

Cada una de estas situaciones puede desarrollarse como estudio de investigación. El nuestro se restringe a las siguientes preguntas:

- ¿Cuál es la concepción de los estudiantes de sexto grado acerca del concepto de multiplicación?

- ¿Qué procesos o interacciones mentales desarrollan los estudiantes de sexto grado para aprender el concepto de multiplicación?

\section{JustifiCACIÓN}

Abordar los procesos cognoscitivos como un sistema aislado de las interacciones educativas, es confiar las transformaciones del conocimiento a la desidia y a la perdida de credibilidad y cientificidad de las corrientes renuentes a estas nuevas tendencias investigativas.

Considerar el pensamiento como algo evolutivo en constante transformación procesual y buscar los procesos de construcción e interpretación de las regularidades naturales y representativas, determinan la importancia del desarrollo de este estudio. 
¿Cuántas veces se ha escuchado: ¿por qué mi hijo no aprende matemáticas? ¡He ensayado todos los métodos para que pueda aprender matemáticas, y aún no lo he logradoi ¿qué le pasa a este niño?. Esta preocupación no es sólo de la madre o padre desconsolado sino también de todos los que de una u otra forma, se encuentran inmersos dentro de la problemática de la enseñanza y el aprendizaje de las matemáticas.

Consciente del problema relacionado con la enseñanza y el aprendizaje de las matemáticas, se necesita explorar las interacciones mentales que realizan los niños para construir y expresar el concepto de multiplicación. El propósito de este estudio consiste en identificar el proceso utilizado para llegar a la transformación de estas preconcepciones sin considerar al estudiante como el único culpable de tan importante problema, sino presentarle el procedimiento aceptado por la comunidad de expertos para llegar a la construcción del conocimiento.

Este proceso debe llevarnos a la reconstrucción de políticas que sean del conocimiento de los administradores de los programas, como los encargados en última instancia de aceptar o rechazar los planteamientos surgidos del estudio. Las recomendaciones, basadas en los hallazgos de este estudio, pueden ser utilizadas para la elaboración de estrategias metodológicas que faciliten la enseñanza y el aprendizaje del concepto de multiplicar.

\section{REVISIÓN DE LA LITERATURA}

La búsqueda de evidencias nos permitió identificar algunos estudios relacionados con este. Lo anterior llevó a los investigadores a realizar un examen de literatura del cual se obtuvieron hallazgos que fundamentan la necesidad de desarrollar esta investigación. Entre los estudios afines a esta investigación se encuentran:

Foye, B.C. y Kamii, C. (1996) quienes manifiestan en su trabajo que los niños que desarrollan la suma requieren de la aplicación de un orden superior de pensamiento para la multiplicación. En esta investigación se entrevistaron individualmente a 136 niños en los grados de 1 ro a 5 to y se utilizaron tareas Piagetanas que permitieron determinar el desarrollo del pensamiento de la suma a la multiplicación. Se encontró que en un $45 \%$ de los estudiantes, el "pensamiento multiplicativo" comienza a aparecer en los inicios del segundo grado y se desarrolla lentamente. Sólo un $48 \%$ de los estudiantes del quinto grado demostró alguna consistencia en el uso del "pensamiento multiplicativo". Foye \& Kamii concluyen que la introducción de la multiplicación en el segundo grado es apropiada, y que los educadores no deben esperar hasta quinto grado para enseñar la multiplicación a todos los niños.

La relación de esta propuesta con el estudio permite consolidar algunos elementos de juicio sobre los cambios y transformaciones que puede sufrir el concepto de multiplicar en la estructura de pensamiento de los alumnos. En otras palabras, se intenta identificar las relaciones entre las interacciones mentales de los alumnos y las acciones que realizan para llegar a la construcción del concepto de multiplicación.

Page, A. (1974) afirma que, de acuerdo con su propia experiencia en el salón de clases, los niños no tienen problemas con el concepto en sí, sino que se 
les hace difícil entender la relación entre el concepto y su representación numérica. Los niños llegan al primer grado con un entendimiento intuitivo de la resta. Una de nuestras tareas, según los estándares de matemáticas, consiste en crear el nexo entre los conocimientos previos del niño y las matemáticas. Es necesario proveer una variedad de situaciones donde se utilice la resta para que el niño pueda ampliar el campo de aplicación de dicha operación matemática. El problema surge cuando le pedimos al niño que establezca entre la situación y una presentación numérica. (Regularmente se olvida del lenguaje el cual es crucial para realiza las conexiones cognitivas). Se necesita ofrecer significados al proceso algorítmico a través del lenguaje.

Este trabajo identifica algunos aspectos de fundamental importancia en las interacciones mentales y están relacionadas con el lenguaje. Se considera que el lenguaje posibilita que el estudiante integre lo teórico con lo operativo en la multiplicación.

Cramer y Karnowski (1995) tratan de descubrir la importancia del lenguaje en las representaciones matemáticas. Basados en el modelo "Lesh Translation Model", los autores enfatizan específicamente la importancia de la comunicación en el aprendizaje de las matemáticas en el proceso de instrucción. El lenguaje ayuda a los niños a construir nexos entre sus experiencias matemáticas informales y los símbolos abstractos utilizados en matemáticas. El lenguaje facilita las conexiones entre las diferentes representaciones matemáticas y las ideas. La escritura sobre las matemáticas ayuda al estudiante a profundizar su entendimiento.

Se puede definir el entendimiento de las matemáticas como la habilidad para representar una idea matemática en di- versas formas y realizar conexiones entre las diferentes representaciones. El lenguaje matemático informal del estudiante es un mediador entre el significado y las diferentes representaciones; adicionalmente, puede suplementar la abstracción de ideas matemáticas de los estudiante.

Kouba y Franklin (1995) concluyen que los niños se desempeñan pobremente en los problemas de multiplicación y división, debido al uso persistente de estrategias inadecuadas para resolver este tipo de problemas matemáticos, reforzados inadvertidamente por una serie de factores. Unos de estos factores reside en que los problemas de multiplicación y división en los grados K-4 se limita a operaciones con números enteros. Esta situación nos ayuda a que los niños puedan percibir la realidad y concluyen "falsamente" que la multiplicación siempre produce números grandes y la división, números pequeños.

Los niños necesitan experiencias con situaciones concretas y que se les guíe y oriente para el entendimiento de modelo a escala (Hndrickson, 1986). Las investigaciones muestran (Kaput, 1989) que los niños aprehenden mejor cuando pueden utilizar diferentes representaciones para las situaciones de multiplicación y división, de manera que puedan expresar las relaciones existentes entre dichas representaciones o modelos. Muchos niños realizan sus primeras relaciones matemáticas de la multiplicación y la división empleando modelos o representaciones relacionadas con la suma y la resta.

Los maestros deben motivar a los estudiantes para que apliquen estrategias que le permitan encontrar sentido con su experiencia cotidiana. Los niños obtienen rendimientos académicos ade- 
cuados cuando pueden desarrollar sus propias historias matemáticas (orales o escritas) de multiplicación o división y utilizar modelos para representar sus historia. Los estudiantes en sus diferentes niveles pueden lograr progresos en el aprovechamiento matemático, si se les permite comparar sus ejecuciones con la de otros estudiantes que estén ubicados en un nivel más avanzado. Esto puede lograrse mediante la enseñanza cooperativa y la planificación de actividades relacionadas con preguntas del medio en secuencias o de actividades de exploración.

Lamper (1989) afirma que ningún algoritmo simple es el "correcto" para enseñarse. Al igual que en la suma y en la resta, cuando se le permite al estudiante crear sus propios algoritmos multidígitos, aumenta la comprensión y la flexibilidad en el uso de las operaciones matemáticas. El niño puede explicar lo que hizo y demostrar la validez de su algoritmo inventado a través de la manipulación de objetos físicos o mediante la creación de arreglos pictóricos. El uso de algoritmos informales tomara más tiempo, sin embargo, el uso de dicho tiempo produce un ahorro de tiempo en la práctica de reglas matemáticas y aumenta la comprensión y la motivación del niño.

Norwood y Carter (1994) describen como los estudiantes, a través de la escritura de un diario, muestran el entendimiento respecto a cualquier concepto matemático. Mediante el diario personal, el estudiante puede demostrar si domina simplemente la parte mecánica de la multiplicación pero no el concepto o si poseen ambos dominios. Este diario también se emplea para repasar o evaluar las ideas de los estudiantes sobre algún tema antes de su introducción. Además, se utiliza como actividad de eva- luación para determinar cuan bien los estudiantes entienden el tema que se esta desarrollando en clase. La estructura de los diarios personales traen a la luz pensamientos y entendimiento en un ambiente típico de salón.

Budget, Turner y Cooney (1996) consideran que para conocer y entender cómo los estudiantes procesan, es necesario observar, escuchar y recopilar evidencia de lo que los estudiantes están aprendiendo. Para lograrlo, es pertinente examinar el pensamiento del estudiante a través de sus interacciones con las tareas.

Esta investigación va dirigida en esa dirección. Se pretende examinar cómo los estudiantes han organizado el concepto de la multiplicación en su estructura de pensamiento. Investigaremos cómo lo construyeron y si pueden expresarlo a través de todo el proceso de aprendizaje cuando se les exige o pide su definición.

Buschman (1995) propone como parte de la enseñanza de las matemáticas, identificar la naturaleza del pensamiento del estudiante y las estrategias utilizadas para solucionar problemas a nivel conceptual. Mediante la interacción y la comunicación, el estudiante puede reflejar su entendimiento matemático, o de las matemáticas, para establecer conexiones e internalizar los conceptos. De esta manera, el estudiante puede recordar, entender, utilizar y descubrir nuevos conceptos. Buschaman también plantea que los estudiantes necesitan tiempo para observar, trabajar con pares y construir un lenguaje matemático propio. Cuando se comparte conocimiento con los pares, el conocimiento adquirido es útil y práctico en la aplicación en diferentes situaciones. Cuando el estudiante utiliza su propio vocabulario, su entendimiento matemático 
es más preciso, emplea palabras aprendidas en situaciones y contextos reales. De esta manera puede entender el significado de cada palabra y la definición del concepto.

De León y Fuenlabrada (1996) en un estudio sobre los procedimientos que utilizan los estudiantes de primaria para la solución de problemas de reparto, encontraron que la mayoría de los niños tiene dificultad para resolver problemas de la selección de pedazos. Los niños de los primeros grados no han construido, en el plano de la acción implícita, la relación de igualdad entre el total de enteros de un reparto y el total de pedazos del mismo reparto; los niños de tercero a sexto que tienen dicha relación, al menos de manera implícita, no logran funcionalizarla con anticipación en el contexto de la selección de pedazo. En síntesis, señalan que la construcción del significado de las fracciones es complejo y prolongado y ello resulta de la interacción de los niños, con situaciones problemáticas, con sus esquemas de conocimiento y con los sistemas de significados o signos.

La revisión de la literatura permite reexaminar este estudio y las concepciones de los estudiantes, como también los referentes teóricos para explicar cómo los estudiantes aprenden los conceptos matemáticos de la multiplicación y las estrategias que emplean para incorporarlos en su estructura de saberes y expresarlos cuando se les solicita.

\section{MARCo teóRICo}

\section{Los niveles energéticos de pensamiento}

El problema concerniente a la enseñanza y el aprendizaje de las ciencias en general, ha sido objeto de un gran número de investigaciones, las cuales en última instancia, han buscado resolver de una u otra forma el paradigma de transmisión-asimilación o de la enseñanza tradicional.

Mancomunadamente, los supuestos teóricos psicológicos y epistemológicos, han hecho sus aportes, tratando de vencer los obstáculos paradigmáticos renuentes a desaparecer del panorama de la investigación educativa. Dentro de estos enfoques teóricos, el de mayor aceptación por la comunidad educativa, en los actuales momentos es la teoría cognoscitiva. Esta enfatiza sobre los niveles y el desarrollo de comprensión y asimilación del aprendizaje de los conceptos científicos.

Enmarcados en esta concepción, se pretende realizar un aporte teórico que permita homologar las actividades mentales como cambios en la estructura de conciencia, con las transiciones energéticas que ocurren en los movimientos naturales. Esta construcción mental la hemos denominado: Los Niveles Energéticos de Pensamiento y surgen como una alternativa para las investigaciones educativas de las ciencias naturales, pues, los intentos de encontrar modelos teóricos que caracterizan o fundamentan los estudios educacionales, en estos momentos es bastante difícil. Este modelo se basa en los espaciamientos de los niveles energéticos que forman el espectro electromagnético, como base teórica fundamental para explicar las rupturas y formación de nuevas estructuras físicas, químicas y biológicas. Estas interacciones son generadas por potenciales energéticos que aumentan el movimiento de las partículas, generando nuevas formas o repre- 
sentaciones que requieren de explicaciones más refinadas de la nueva sustancia, fenómeno, situaciones o suceso.

De igual manera, se puede afirmar que la estructura de conciencia de los humanos, está constituida por niveles energéticos de pensamiento. Para que una persona pueda transferir o transformar un cons-tructo, requiere de potenciales intelectivos que le permitan establecer relaciones y saltos cualitativos, dentro de los niveles energéticos de pensamiento de su macro estructura evolutiva. En otras palabras, para que un individuo pueda pasar de una hipótesis o conjetura a un concepto más refinado y aceptado por la comunidad científica, se requiere que las interacciones energéticas de pensamiento permitan estimular los niveles jerárquicos de la estructura mental, producto de la distribuciones energéticas entre los niveles de aprendizaje, que se corresponden con los diferentes movimientos espectrales presente en la estructura cognitiva.

Puede decirse que todos estos cambios energéticos se suceden de idéntica forma en la estructura de pensamiento. Por la adición constante de nuevos potenciales de saber que permiten establecer acoplamientos o combinaciones, entre un nivel de aprendizaje general y uno específico, aumentando en consecuencia los saltos de aprendizaje de un nivel a otro.

\section{Metodología}

Esta investigación es un estudio de caso de tipo descriptivo que busca explorar las interacciones mentales de los estudiantes de sexto grado, de la escuela intermedia, para definir y aprehender el concepto de multiplicación. La interpre- tación de los datos obtenidos será de tipo cualitativo, ya que no se medirán variables en términos numéricos. Se hará una interpretación de los protocolos grabados, resultados de entrevista con preguntas "reconciliadoras".

Este estudio se llevó a cabo con estudiantes del Colegio Congregación Mita, en Hato Rey de la escuela intermedia Raúl Ybarra del Departamento de Educación, en el pueblo de Maricao. La muestra la constituyó un grupo de ocho (8) estudiantes, de las clases de matemáticas, seleccionados por disponibilidad y conveniencia.

\section{EtAPAS DEL PROYECTO}

\section{Preparación del proyecto de estudio}

Consiste en la preparación y redacción detallada de los diferentes elementos conceptuales que integran la propuesta. Permiten brindar una visión general de lo que se pretende desarrollar en el proceso de investigación.

\section{Elaboración del instrumento de investigación}

La técnica utilizada para la recolección de datos fue la entrevista con preguntas "reconciliadora" mediante la cual se pretendía detectar las interacciones mentales desarrolladas por los estudiantes desde sus primeros contactos con el concepto de multiplicación, su evolución, cambios aparentes o regreso a su estado inicial. Es decir, identificar las transformaciones conceptuales generadas en la estructura de conciencia, a través de los saltos intelectivos de los niveles energéticos de pensamiento que 
se producen con la formulación de las preguntas "reconciliadoras".

a) $\mathrm{N} 1$ Avance N2 Nivel inicial de la estructura de pensamiento

Nivel de reconciliación y transformación de la nueva información con la existente en la estructura mental

b) $\mathrm{N} 1$ Retorno N2

Transición parcial por algunos $y$ antiguos elementos de la estructura de pensamiento

\section{Entrevista con preguntas reconciliadoras}

La entrevista busca identificar en los estudiantes el desdoblamiento conceptual progresivo. Entendemos por ese proceso la transformación conceptual continua en los seres humanos a través del contacto directo o indirecto con el conocimiento cotidiano o científico. En otras palabras, son los cambios paradigmático los que permiten cambiar o modificar una determinada concepción de un evento o fenómeno por otro más acabado y aceptado por la comunidad de especialista en estos temas. Adicionalmente, en ese proceso, toda persona se ve abocada al desarrollo intelectivo, a superar, recordar o mantener las transiciones e interacciones conceptuales que se dan ante nuevas situaciones, hechos o fenómenos del ambiente natural, social y cultural, en el cual se desarrollan periódica o perentoriamente los proceso de construcción del conocimiento.

Aunque no es una entrevista estructurada, presenta un orden secuencial para obtener los logros propuestos. Este orden secuencial no permite divagar a partir de las repuestas de los entrevistados lo cual conlleva a contradicciones conceptuales por los implicados en el estudio (entrevistador/entrevistado). Aunque se abre la posibilidad, a través de las preguntas "reconciliadoras" que el estudiante construya o negocie transitoriamente su concepción por la que surja en el diálogo.

La entrevista con preguntas "reconciliadoras" constituye una alternativa para la caracterización de los procesos mentales, que busca identificar las transiciones cognitivas de los individuos cuando son expuestos a un recorrido histórico-social-cultural del conocimiento en un área de saberes específicos.

La entrevista se inicia con varias preguntas dialógicas que permiten al entrevistado (profesor o estudiante), identificar o familiarizarse sobre el tema que deseamos indagar, son de carácter directo y de ellas, podemos determinar el desarrollo y los logros alcanzados al final de la entrevista.

\section{Guía PARA LA ENTREVISTA}

\section{RECONCILIADORA}

Como parte del estudio de las Ciencias Básica (Biología, Química, Física y Matemáticas) son muchos los conceptos, ideas, términos o palabras que debes aprender para poder comprender e interpretar un fenómeno natural o matemático.

¿Puedes mencionarme algunos de estos conceptos, ideas, términos o palabras que recuerdes te causaron problemas $o$ dificultad para aprenderlos? ¿Por qué?

¿Qué piensas de esos conceptos, ideas, términos o palabras? 
¿Puedes explicarme cómo hiciste para entender y aprenderte esos conceptos, ideas, términos o palabras? ¿Los memorizaste? ¿Por repetición? ¿Por demostración o solamente con la explicación del profesor?

Quiero que hablemos con relación al aprendizaje de estos conceptos (ideas, términos o palabras), específicamente sobre un concepto de matemática que tú conoces y del cual te has valido, o haz empleado para resolver algunos problemas. El concepto (idea, términos o palabras), al cual quiero referirme es el de la multiplicación. Es preciso mencionarte que nadie sabrá tu nombre, por eso esperamos que puedas decirnos espontánea cómo te enseñaron y cómo aprendiste el concepto (idea, término o palabras) de multiplicar.

En los años de estudios cursados y basándote en tus experiencia, ¿Puedes decirme qué entiendes por multiplicación? ¿Tienes otra forma de definirlo o explicarlo?

¿Cuál fue la estrategia, mecanismo o medios que utilizaste para aprender el concepto de la multiplicación?.

¿En qué otros conceptos matemáticos te apoyas o basas para entender y aprender el concepto de multiplicación? ¿Por qué?

¿Por qué crees que estos conceptos, en los cuales te apoyaste para explicar o definir el concepto de multiplicación, son importantes?

Estos conceptos, términos, ideas o expresiones que acabas de mencionarme, ¿los aprendiste de la misma forma como aprendiste el concepto de multiplicar? ¿Te fueron más fáciles o más difíciles de aprender?
¿Recuerdas cuál era tu idea sobre el concepto de multiplicar antes que el profesor te lo explicara? ¿Por qué?

¿Puedes ofrecerme ejemplos de situaciones donde tengas que utilizar (aplicar) la multiplicación?

\section{Hallazgos del estudio}

\section{Entrevista con preguntas "reconciliadoras"}

Compartir con estudiantes de sexto grado respecto a sus experiencias en el proceso de enseñanza y aprendizaje ofrece la oportunidad para conocer mejor sus fortalezas y debilidades en las diferentes áreas académicas. Por lo general, el estudiante presenta opiniones divididas respecto a su satisfacción y comprensión de la materia, así como su concepción de los diferentes términos (conceptos) que facilitan el estudio de ésta.

En su gran mayoría, los estudiantes manifiestan comprender los términos relacionados con su materia. Aceptan el reto de definirlos según su entendimiento y según han sido definidos por sus maestros o de otras personas de las cuales adquieren las primeras definiciones y aplicaciones del concepto.

Con respecto a las matemáticas, muchos estudiantes indican estar "bien" en la clase, con el único agravante de que la multiplicación y la división así como las fracciones constituyen los conceptos de mayor dificultad. En este estudio preferimos prestarle especial atención a la multiplicación. Según se desprende de los datos ofrecidos por los mismos estudiantes:

a.-Entienden lo que el maestro presenta en sus clases si es explícito y creativo. 
b.-Los conceptos que más dificultad causan en los estudiantes son las fracciones, la multiplicación, división y las tablas de multiplicar.

c.- Todos tienden a definir la multiplicación operacionalmente como la multiplicación de un número por otro, el número de veces que se quiera, es una suma repetida o abreviada de algún número, y no muestran otra forma de definirla.

d.- Utilizan el algoritmo tradicional para realizar los ejercicios.

e.- Se basan en la tabla de multiplicar ya memorizada, uso de los dedos (como manipulativo digital) y la suma para llegar al resultado.

\section{f.- La práctica garantiza el aprendizaje} del concepto (memorización).

g.- Los estudiantes desconocen la jerga técnica, pero operan y resuelven los problemas con fluidez.

h.- La forma vertical de agrupar los factores es memorizada y preferida sobre la horizontal.

i.- Cuando existen dudas se recurre al "experto" para resolver el ejercicioy lograr el éxito (técnica tutorial).

j.-Pueden relacionar su aprendizaje con experiencias pasadas en grados anteriores.

k.- Pueden hacer conexiones (arquitectura y ciencias) y aplicar el concepto a situaciones cotidianas (en el supermercado, en la tienda de ropa, transacciones de dinero, labor diarias como carpintero y otras).

I.- En algunos casos, la matemáticas desarrollan fobia hacía ésta.
m.-Se recomienda el uso de calculadora como solución inmediata a la dificultad o falta de conocimiento; papel y lápiz, cuando sea necesario.

\section{Discusión Y CONCLUSIONES}

Una vez redactadas las entrevistas reconciliadoras de los estudiantes de sexto grado sobre las concepciones del concepto de multiplicar, se procedió a establecer categorías que nos permitieran identificar, describir, analizar e interpretar, las posibles similitudes y diferencias en el contexto de las entrevistas efectuadas. Identificamos que las concepciones que los alumnos entrevistados poseen sobre el concepto de multiplicar, así como los elementos específicos relacionadas con este concepto, no sólo no se diferencian de las concepciones aceptadas por la comunidad de expertos, sino también de la propia perspectiva que en los niveles de enseñanza se tratan de impartir. La necesidad de aprender y darle explicación a un hecho, acontecimiento o fenómeno, genera en cada individuo unos elementos simbólicos que la permiten comprender e interpretar estas situaciones, de acuerdo con sus capacidades mentales, producto de sus interacciones en el medio.

Una de las dificultades que presentan los estudiantes entrevistados sobre el concepto de multiplicar reside en la escasez de elementos teóricos que le faciliten una explicación más acabada de la construcción del constructo. Como producto de esta caracterización, encontramos que mientras estos estudiantes no tuviesen papel y lápiz para detallar el proceso operativo del algoritmo de la multiplicación, no presentaban ni siquiera una idea de lo que era el concepto de multiplicar. Para este grupo de 
estudiantes, las operación de multiplicar es totalmente manipulable, demostrativa, repetitiva, memorística a través de la suma abreviada, careciendo sus procesos mentales de todo sentido de formalidad y de abstracción.

Consideran el significado del concepto de multiplicar como replicar y replicar como repetición. Los estudiantes entrevistados, una vez tiene a la mano lápiz y papel, cuando se les indagaba sobre qué se entendían por multiplicar, muchos lo expresaban como la repetición de un número, aunque su explicación conceptual dejase mucho que desear sobre su coherencia. Algunos expresaron:

(1) ..."yo entiendo que multiplicar es coger ese número y hacerlo otra vez, otra vez, y después sumarlo", (2) ..."pues, que tú multiplicas los números por otro ...y entonces te da el resultado", (3) ..."la repetición de un número...varias veces, las veces que te indiquen", (4)..."pues, eso son...fracciones de números que tú los tienes que multiplicar con otros y cuando los multiplicas te da un resultado".

Esta última definición expresa mayor complejidad que las anteriores, pues, introduce elementos conceptuales como son las fracciones y los resultados que hacen más completa la expresión del concepto de multiplicar. Otros lo expresan como una situación preconcebida de causa-efecto, en la cual la manipulación de unos símbolos numéricos proporcionan un resultado cuando dicen:

..."mira, estamos multiplicando por esto y te da esto, esto es lo que significa multiplicar", o cuando dicen "multiplicar es añadir más a un número", también lo expresan como algo aumentativo en el caso: .."algo que tú multiplicas o lo añades o lo agrandas, es decir, añadir varias veces, repetir el mismo número o sumarlo".
Aunque los entrevistados utilizaron papel y lápiz para expresar el concepto de multiplicar, algunos lo identificaron totalmente como un proceso operativo al expresar "multiplicar":

... " es como decir tres por cuatro, que es lo mismo que cuatro por tres.. cuento tres más tres igual a seis, más tres igual a nueve, más tres igual a doce". Este ejemplo esta intrínsicamente relacionado con el algoritmo tradicional de la forma operativa de resolver la multiplicación.

También la definen como "sumar $x$ número por $x$ veces", el cual manifiestan que es la única forma que tienen para definirlos. Es necesario destacar que sólo un estudiante logra definir el concepto de multiplicar como una suma abreviada al expresar:..."es sumar un número varias veces para llegar a la contestación ...el número de veces que te diga la multiplicación".

Con relación al proceso de aprendizaje y la comprensión de la multiplicación, estos elementos están influenciados por la aprehensión memorística, por la repetición oral y escrita, la tutoría de expertos y la técnica de relajación musical (música-terapia). Esta influencia se manifiesta en las expresiones, de la siguiente manera:

... " sé multiplicar porque sé las tablas de memoria, pues cogía ese número y si me pedía dos por seis, pues ponía el dos, seis veces y después cogía un papel y lo sumaba. Después cogia todos los números de la tabla del seis y lo hacía así: seis por uno, ponía el seis una vez; seis por dos, ponía el seis dos veces, después tres veces y lo sumaba, y así, hasta terminar la tabla del seis. Después lo repetía muchas veces y el total me lo aprendía". Otro estudiante expresa que, "cuando estaba en cuarto (se refiere al grado), la maestra tenía 
unos cassette con música de rap, entonces yo, por las noches me ponía a oír esos cassette, a practicar las tablas hasta que me las aprendí. Las estudié y las estudié y cuando mi mamá me preguntó todas las tablas me las sabía, que hasta en el carro me las preguntaba: ¿cuánto es esto por esto? Y ¿esto por esto?, hasta que las memorice”.

Al preguntarles si aplicaban otra estrategia para aprender a multiplicar, una estudiante respondio:

"cojo un papel, pongo un ejercicio de multiplicar, que a veces te salen tres arriba y tres abajo, entonces yo no entendía bien eso. La maestra me explicó que el primero se multiplica por los tres de arriba y después el segundo es lo mismo y el tercero es lo mismo. Yo lo que hacía era que multiplicaba los números en línea y los sacaba mal"

(Se le pide a la estudiante que presente ejemplo de un ejercicio por escrito para que explique qué era lo que hacía). La estudiante escribe: 897 X 123 y explica: "yo lo que hacía era que multiplicaba $3 \times 7,2 \times 9$ y $1 \times 8$. Luego, la maestra me explicó que se multiplicaba el tres por cada uno, $3 \times 7$ luego $3 \times 9$ y $3 \times 8$, y lo mismo hago con el dos por cada uno de los números y lo mismo con el uno".

En esta estudiante se observa una deficiencia en el dominio algorítmico, y su aprendizaje se realiza por demostración, es decir, esta estudiante presentaba confusiones entre la suma y multiplicación en su forma operativa.

Todos los estudiantes entrevistados utilizan los dedos como manipulativo digital para el desarrollar el proceso operativo de la multiplicación. También, el elemento conceptual para la construcción del concepto de multiplicar es la suma. El aprendizaje del concepto de multiplicar lo construyen practicando, en forma oral y escrita, las tablas de multiplicar. Su proceso mental está basado en la repetición mental y escrita de la multiplicación cuando dicen:

"yo aprendí a multiplicar practicándolas y escribiéndolas y diciéndomelas un montón de veces hasta que me las aprendi", otro dice: "mi mamá me ayudaba, ella me preguntaba y yo tenía que contestar y me hacía repetirlas muchas veces".

Lo anterior indica que los estudiante necesitan de la guía de un adulto, experto o compañero de un nivel más avanzado para el aprendizaje de la multiplicación. Vy-gotski afirma que la zona de desarrollo próximo es la distancia entre el nivel real de desarrollo, determinado por la capacidad de resolver independientemente un problema, y el nivel de desarrollo potencial, determinado a través de la resolución de problemas bajo la guía de un adulto o en colaboración con otro compañero más capaz.

En el proceso operativo de la multiplicación, todos los entrevistados emplearon la forma algorítmica tradicional, aunque aplicando algunas técnicas enseñadas por los profesores para facilitar la consecución de los resultados. Ejemplo de estas técnicas es el método del asterisco: colocan un asterisco en el espacio vacío al realizar una multiplicación con más de dos multiplicadores y multiplicandos. Es decir, sólo conocen una sola forma algorítmica de plantear la solución de la multiplicación. Al realizar la multiplicación, ninguno de los entrevistados mencionó los factores o elementos de la multiplicación, todos emplearon el recurso de las tablas de multiplicar escritas porque dudaban y no las habían memorizado. Todo esto nos lleva a pensar que la multiplicación es enseñada en forma memorística y repetitiva y la información se presenta directamente al alumno, sin ofrecer alternativas que produzcan en él interac- 
ciones que puedan cambiar su estructura de pensamiento, como son la resolución de la multiplicación a través de las propiedades de ésta o mediante la solución por analogías.

Podemos plantear que el concepto de multiplicar es construido por los alumnos, a través de las experiencias sucesivas a las que se ve expuesto, producto de la repetición y la memorización, como el manejo manipulativo digital, que va desarrollando a lo largo de su contacto con el ambiente escolar, familiar y social, y no como una entidad independiente y definida de la actividad mental. El proceso de comprensión del concepto se lleva a cabo a través de la interacción del estudiante con el medio.

Otras manifestaciones que logramos identificar es lo referente al proceso mental que estos estudiantes aplican para mantener estos constructos en su estructura de conciencia: es decir, la organización operativa de estos conceptos en términos de la relación entre la suma y la multiplicación. Para estos alumnos el conocimiento debe estar organizado para que pueda memorizar y ser asimilado, en otras palabras, ofrecerlos en "paquetes" que permitan establecer jerarquías en la estructura de pensamiento. La otra manifestación es que no conservan este constructo en la mente mediante la racionalidad, sino a través de la memorización y la repetición.

Los niveles energéticos de pensamiento para este grupo de alumnos son impredecibles, ya que los saltos cuantizados de conocimientos, según sus expresiones conceptuales, se mantienen latente en la estructura de conciencia en estos sujetos, a pesar de que duran- te la entrevista dejan entrever cambios que parecen ser producto de las interacciones conceptuales con los temas matemáticos cursados.

\section{Conclusiones}

- Los estudiantes participantes de este estudio parecen poseer escasos elementos teóricos para explicar el concepto de multiplicar, debido al aprendizaje memorístico y repetitivo, y por el uso de manipulativos y demostraciones totalmente concretas.

- La definición del concepto de multiplicar es expresado en forma operativa, mediante la repetición mental y escrita.

- El elemento conceptual y operativo para la construcción del concepto de multiplicar es la suma.

- No expresan los factores o elementos de la multiplicación (multiplicador, multiplicando y resultado) como unidades esenciales del carácter operativo de la multiplicación.

- No utilizan las propiedades de la multiplicación (asociativa, conmutativa, elemento neutro y distributiva) para plantear otras alternativas de solución al algoritmo tradicional que realizan.

- Los niveles energéticos de pensamiento son imprescindibles para el carácter operativo y mecánico que suelen atribuir a los principios y modelos que utilizan para explicar el concepto de multiplicar.

- La observación es básica dentro de los procesos mentales, pero no determinante. El conocimiento no se adquiere por una internalización de un significado 
exterior ya dado, sino por la construcción desde dentro, de representaciones e interpretaciones adecuadas.

- La principal dificultad para la correcta adquisición del concepto de multiplicar no está en la existencia de preconcepciones de estos alumnos, sino más bien en los métodos aplicados.

\section{REFERENCIAS BIBLIOGRÁFICAS}

BRIDGET,A., TURNER, P. and COONEY, T. 1996. Analyzing Teaching and Learning: The art listening. The Mathemaics Teacher. 89 (4), 326329.

BUSCHMAN, L. 1995. Communicating in the language of Mathematics. Teaching Children Mathematics. 1(16), 324-329.

CHANCELOR, D. 1991. High order thinking: A basic skill for everyone. Arithmetic Teacher, 38 (6), 48-50.

CORREA, a. 1991. El pensamiento crítico: Una alternativa a la excelencia en la enseñanza matemáticas. Ponencia presentada en la Conferencia Regional del Sureste del Concilio Nacional de Maestros de Matemáticas, San Juan, P.R.

CRAMER, K. and KARNOWSKI, L. 1995. The importance of informal language in representing mathematical ideas. Teaching Children Mathematics. 1 (6), 332-335.

DE LEÓN, H. and Fuenlabrada, I. 1996. Procedimientos de la solución de niños de primaria en problemas de reparto. Revista Mexicana de Investigación Educativa. 1 (2), 268-282.
FAYE, B. C. and KAMII, C. 1996. Identification of multiplicative thinking in children in grades 1 5. Journal for Resarch Mathematics Education, 27 (1), 41-51.

FIGUEROA, R. 1995. Los niveles energéticos de aprendizaje: Una alternativa teórica en la investigación educativa de las ciencias naturales. Seminario Internacional Calidad en la Enseñanza de las Naturales. Junio 28 julio 01. Universidad delAttántico, Barranquilla. Colombia.

KOUBA, V. L. and Franklin, K. 1995. Multiplication and division: Sense making and meaning. Teaching Children Mathematics, 1 (9), 574-577.

KOUBA, V. L. and Franklin, K. 1995. Language, intellectual structure and common mathematical errors: A call for research (May 1994). School Science and Mathematics, 9 (5), 235-239.

NORWOOD, K. S. And CARTER, G. 1994. Journal Writing: An insight into students understanding. Teaching Children Mahematics. 1(3), 146-148.

PAGE, A. 1994. Helping students understand subtraction. Teaching Children Mahematics. 1 (3), 140-143.

SIMON, M. A. and BLUME, G. W. 1994. Bulding and understanding multiplicative relationships: A study of perspective elementary teachers. Journal for Research in Mathematics Education, 25 (5), 472-493.

WILSON, Melvin R. 1994. One preservice secondary teacher's understending of function: The impact of a course integratingmathematical content and pedagogy. Journal for Research in Mathematics Education, 25 (4), 346-370. 